\title{
Screening of toxicological properties of 4-methylbenzoic acid by oral administration to rats
}

\author{
Mariko Shirota, Takayuki Seki, Kazumi Tago, Hiroyasu Katoh, Hideki Marumo, \\ Mami Furuya, Tomoko Shindo and Hiroshi Ono
}

Hatano Research Institute, Food and Drug Safety Center, 729-5 Ochiai, Hadano, Kanagawa 257-8523, Japan

(Received April 3, 2008; Accepted May 26, 2008)

\begin{abstract}
Oral toxicity of 4-methylbenzoic acid in male and female Sprague-Dawley rats was profiled through a twenty-eight-day repeated dose toxicity study (the 28-day study) and a screening test for reproductive/developmental toxicities (the reproduction/developmental study) conducted under Organisation for Economic Co-operation and Development (OECD) test guidelines. Daily administration of 4methylbenzoic acid, at a dose level of $0,100,300$ or $1,000 \mathrm{mg} / \mathrm{kg}$, did not show any adverse effect on reproductive organs of animals in the 28-day study. In the reproductive/developmental study, however, $1,000 \mathrm{mg} / \mathrm{kg} /$ day of the compound reduced epididymal weights and increased incidence of cauda epididymal oligo/azoospermia. While the compound did not affect estrous cycle or mating performances, 1,000 $\mathrm{mg} / \mathrm{kg}$ of the compound reduced fertility. Furthermore, $300 \mathrm{mg} / \mathrm{kg}$ or more of the compound increased pre-implantation loss, which resulted in a decrease in the number of offspring, and reduced body weight gain of the dams during the latter period of gestation. From these results, the no-observed-effect-level (NOEL) for reproductive/developmental toxicities is considered to be $100 \mathrm{mg} / \mathrm{kg}$, whereas $1,000 \mathrm{mg} / \mathrm{kg}$ did not show any effect on neonates. In the 28 -day study, NOEL is considered to be $300 \mathrm{mg} / \mathrm{kg}$ for male and female rats, since $1,000 \mathrm{mg} / \mathrm{kg}$ of the compound caused, in both sexes, a few minor changes, such as temporal salivation, a slight increase in food consumption and a moderate increase in blood aspartate aminotransferase (AST) activity. Thus, 4-methylbenzoic acid has the potential for reproductive toxicity, with diverse adverse effects on the epididymis, after repeated administration, observed in the two studies.
\end{abstract}

Key words: 4-methylbenzoic acid, Epididymis, OECD test guideline, Reproductive toxicant, Repeated dose toxicity, Rats

\section{INTRODUCTION}

Faced with an enormous number of existing chemicals that lacked hazard information, the Organisation for Economic Co-operation and Development (OECD) decided, in 1990, to undertake an investigation of such chemicals in cooperation with its member countries. They gave priority to high production volume (HPV) chemicals in collecting the data for an initial assessment of the hazard. A project was launched in 1990 to complete the dossiers of screening information data sets (SIDS) of toxicity for HPV chemicals through testing, and the work has been done cooperatively in Japan, as well as in other countries. Since 1991, we have participated in the testing under the auspices of the Ministry of Health (, Labour) and Welfare.

4-Methylbenzoic acid ( $p$-toluic acid) was selected from the OECD List of HPVs (OECD, 2004). 4-Methylbenzoic acid is produced at levels greater than 1,000 tones per year and is used in the manufacture of dye stuffs, colorants and paints; agrochemicals; and anticorrosive additives, as well as being an intermediate in the manufacture of polyethylene terephthalate. Although oral $\mathrm{LD}_{50}$ values in rats and mice have been reported as $3,113 \mathrm{mg} / \mathrm{kg}$ and $2,115 \mathrm{mg} / \mathrm{kg}$, respectively (Mineshita et al., 1978), its toxicological properties are little known. The present study was performed to profile the oral toxicity of 4-methylbenzoic acid according to the standard protocols, "Repeated Dose 28-day Oral Toxicity Study" (the 28-day study) and "Reproduction/Developmental Toxicity Screening Test" (the reproduction/developmental study), in rodents (OECD, 1997a, 1997b). 


\section{MATERIALS AND METHODS}

\section{Test substance}

4-Methylbenzoic acid (CAS No. 99-94-5, purity $98.95 \%$ ) was supplied by Toray (Tokyo, Japan), and was kept at room temperature until use. To prepare dosing samples, the compound was finely ground in a mortar, at first. Then, the ground compound for each dose was suspended in a $0.5 \%$ sodium carboxymethylcellulose solution, and each dose was adjusted to a constant volume of $5 \mathrm{ml} / \mathrm{kg}$. The stability of the suspended compound in the vehicle and the content and uniformity of the compound of each dose were confirmed in Hatano Research Institute.

The doses used in the studies were determined based on a preliminary, 7-day, repeated dose, oral toxicity study of the compound, in which a dose level of $1,000 \mathrm{mg} / \mathrm{kg}$ did not show any toxic effects. This was considered sufficient for the highest dose, and the doses used in the present studies were set at $0,100,300$ and $1,000 \mathrm{mg} / \mathrm{kg}$.

\section{Animals}

Male and female rats of the Sprague-Dawley (Crj: $\mathrm{CD}(\mathrm{SD})$ ) strain were purchased from Charles River Japan (Atsugi Breeding Center, Atsugi, Kanagawa, Japan). For the 28-day study, the animals were purchased at 4 weeks of age, and after an 8-day quarantine period, the animals were divided into 4 groups of each sex according to a stratified allocation based on body weight measured on the day before initial dosing. For the reproduction/developmental study, the animals were purchased at 7 weeks of age and quarantined for 7 days. After the quarantine period, the female rats were monitored for estrous cycle by observing daily vaginal smears for 2 weeks. At 10 weeks of age, the females revolving on a regular 4-day estrous cycle and the males were divided into each 4 groups by the same method as used in the 28-day study.

These animals were kept individually in metallic cages with metal-meshed floors, except copulated females in the reproduction and developmental study, which were kept in flat-bottomed plastic cages with bedding materials (Paper Clean, Nippon SLC, Hamamatsu, Japan) from 18 days after copulation. The animal rooms were maintained with a light-dark cycle of 12-hr (lights on $7 \mathrm{hr}$ ), and temperature and relative humidity were controlled to 21.0 $25.0^{\circ} \mathrm{C}$ and to $40.0-75.0 \%$, respectively, with air ventilation at 15 complete changes per hour. The animals were supplied with solid rodent chow (CE-2, CLEA Japan, Tokyo, Japan) and tap water, ad libitum.

\section{Experimental design}

All procedures described here were approved by the Committee on Animal Experiments of Hatano Research Institute, Food and Drug Safety Center.

The 28-day study

Groups given 0 and 1,000 mg/kg of 4-methylbenzoic acid comprised 10 animals of each sex, including 5 animals of each sex for the 2-week recovery study after the 28-day administration of the compound. The groups given 100 and $300 \mathrm{mg} / \mathrm{kg}$ comprised 5 animals of each sex.

Administration was begun at 5 weeks of age, and the initial day of the administration was designated as Day 1 of treatment. All the animals received daily administration of the compound by gavage for 28 days at a fixed time every day, and the initial day of recovery was designated as Day 1 of recovery.

Signs of toxicity were daily observed. Detailed clinical observations were made as specified in the OECD test guideline 407 (OECD, 1997a) in all animals prior to the initial administration, and once a week thereafter until the end of the recovery period. The observers were unaware of the treatment of each animal. Findings were recorded using a scoring system. In addition to the detailed observation of clinical signs, a 4-item neurobehavioral test battery assessing auditory and visual functions was administered at the last week of treatment.

Body weight was measured 3 times during the first week of the treatment, and twice a week thereafter. Food consumption was determined weekly.

Urinalysis was performed at the final week of treatment and at the final week of the recovery study. Urine of all animals was collected for 4 and $24 \mathrm{hr}$ in a metabolic cage, and was examined for $\mathrm{pH}$, occult blood, protein, ketone bodies, urobilinogen and bilirubin, semi-quantitatively using a urine test strip analyzer (Clinitek 200+, Bayer Medical, Tokyo, Japan), and for its color, turbidity and sediments. The volume and weight of the 24-hr urine was measured, and the specific gravity of the urine was calculated.

Necropsy of the animals was performed after 18-24 $\mathrm{hr}$ of fasting on the day following final treatment and on the day following the recovery period. Under anesthesia with pentobarbital sodium, blood samples were collected from the abdominal caval vein by syringe, with sodium citrate as an anticoagulant, for determination of coagulation times; with EDTA-2K potassium, for hematological examination; and with heparin, for blood chemical examination. Then, the animals were killed by exsanguination from the axillary artery. After gross observation, dissected organs, such as brain, thymus, heart, liver, kidneys, spleen, testes, adrenals and epididymides, were weighed. 
In addition to these organs, spinal cord, lungs, bronchi, stomach, ileum, colon, seminal vesicles, ovaries, uterus, vagina, urinary bladder, thyroid gland, femoral marrow, mesenteric lymph nodes, mandibular lymph nodes and ischiadic nerves were dissected out and fixed in buffered formalin for histopathological examination. The testes were fixed in Bouin's solution, with post fixation in buffered formalin. These organs/tissues were then processed for paraffin embedded block, and sections cut from the blocks were stained with hematoxylin-eosin (HE).

Hematological examination was carried out using automatic blood analysis apparatus (CELL-DYN3500SL, Abbot Diagnostics, IL, USA) for cell counts (erythrocytes (RBCs), leucocytes (WBCs) and platelets), hemoglobin concentration and differential WBC counts. Hematocrit, mean concentration of hemoglobin in the RBC (MCHC) and mean content of hemoglobin in the RBC were calculated. The prothrombin time (PT) and activated partial thromboplastin time were measured using a fully automatic analyzer for blood coagulation (CA-1000, Toa Medical Electronics, Saitama, Japan).

In the blood chemical examination, plasma concentrations of total protein, albumin, total cholesterol, glucose, blood urea nitrogen, creatinine, triglyceride, total bilirubin, inorganic phosphorus and calcium and activities of aspartate aminotransferase (AST), alanine aminotransferase (ALT), alkaline phosphatase (ALP) and $\gamma$-glutamyl transpeptidase (GTP) were determined using a centrifugal automatic blood chemical analyzer (COBAS-FARA, Roche Diagnostics, Basel, Switzerland). Ratios of albumin to globulin were calculated, and plasma concentrations of sodium and potassium were measured using an automated electrolyte analyzer (EA05, A \& T, Yokohama, Japan).

The reproduction/developmental study

The study consisted of 4 dosing groups, and each group comprised 13 males and 13 females. Administration was begun at 10 weeks of age, 2 weeks prior to mating in both, males and females, and was performed by gavage at a fixed time every day. The initial day of the administration was designated as Day 1 of treatment. Mating was performed by co-housing one male and one female from the same treatment group for a maximum of 2 weeks and was confirmed by observation of a copulatory plug or by the presence of sperm in a vaginal specimen. After confirmation of mating, each female was housed individually, and the day on which mating was confirmed was designated as Day 0 of gestation. Administration was continued during the mating period and gestation period, until 3 days after delivery for females. For males, the administration was continued through and after the mating period, for a total of 42 days. For females that had copulated but did not deliver or for females that had not copulated, the administration was continued until 25 days after copulation or to Day 52 of treatment, respectively.

Clinical conditions were observed daily, and body weights and food consumptions were measured weekly. After confirming copulation, the females were weighed on Days 0, 7, 14 and 20 of gestation, and their food consumptions were determined on Days 0-1, 7-8, 14-15 and 20-21 of gestation. When the females delivered live fetuses, they were weighed on Days 0 (the day of delivery) and 4 of lactation, and their food consumptions were determined on Day 3-4 of lactation.

The estrous cycle was monitored daily until copulation. The females that had copulated were allowed to deliver spontaneously and to nurse their own pups until Day 4 of lactation. During the lactation period, the number and sex of dead and live pups were recorded for each dam, and the external morphology and general condition of the live pups were examined daily. The dead pups were examined for external and visceral abnormalities, when possible.

All the males were killed for necropsy on the day after Day 42 of treatment by exsanguination under sodium pentobarbital anesthesia. Maternal animals were similarly killed on Day 4 of lactation, while their offspring were killed for necropsy on the same day by ether inhalation. The females that had not copulated during the mating period and the females that had copulated but did not deliver fetuses were also similarly killed.

At necropsy, males were examined grossly for abnormalities, and the testes and epididymides were dissected, weighed and fixed in Bouin's solution, with post fixation with a buffered formalin solution for processing for histopathological examination. The other reproductive organs, such as ventral prostate and seminal vesicles with coagulating glands, were also dissected and fixed in a buffered formalin solution for histopathological examination. Females were examined grossly for abnormalities. Organs with lesions were dissected for histopathological examination. Ovaries, uterus and vagina from dams were dissected to determine the number of corpora lutea and implantation sites. Then, these organs were processed for histopathological examination. The absence of implantation sites in the uterus of females that had not copulated and females that had copulated but did not deliver fetuses was confirmed under a stereomicroscope. Live pups were euthanized by ether inhalation and were examined for of external and visceral abnormalities.

\section{Statistical analysis}

Fisher's direct probability test was applied to analysis 
of the incidence of animals in which the estrous cycle was altered, the copulation rate, the fertility index, the incidence of offspring with morphological abnormalities, and the incidence of histopathological findings. Graded findings in the histopathological examination were analyzed using the Mann-Whitney U-test. Data from the urinalysis urine quality test were analyzed using a chi-square test.

The other data were analyzed with multiple comparisons when comparing the data from more than 2 groups with those from the control. When comparing the data from a single group with those from the control, Student's t-test or Aspin-Welch's t-test was applied, following an Ftest. In the multiple comparisons, an analysis of variance (ANOVA) test or Kruskal-Wallis's rank test was applied, following examination of the uniformity of variations by Bartlett's method. Significant differences from the control were determined by Dunnett's test. A $p$ value of less than $0.5 \%$ was judged a significant difference.

\section{RESULTS}

\section{Effects of repeated dosing in the 28-day study}

Neither, death nor moribund condition, was observed in any animal, but a few male and female animals in the $1,000 \mathrm{mg} / \mathrm{kg}$ group showed temporary salivation after dosing. Except that, there were no clinical signs related to the treatment, and the scores of the detailed observation of clinical signs in the compound treated groups were comparable to those in the control (data not shown). In addition, no abnormalities were observed in the neurobehavioral test at the last week of treatment (data not shown).

Changes in body weight and food consumption are illustrated in Figs. 1 and 2, respectively. While body weights of males and females in the compound treated groups changed similarly to those in the control group at any period, food consumption of females in the $1,000 \mathrm{mg} /$ $\mathrm{kg}$ treated group was slightly greater than in the control group, from Day 7 to 8 of treatment. Whereas the food consumption of females in this group tended to be greater thereafter, until the end of the treatment, no differences in food consumption were observed in males among any groups throughout the study.

As summarized in Table 1, urine volume measured on the Day 23 of treatment was larger in the males given $300 \mathrm{mg} / \mathrm{kg}$ or more of the compound and in females given $1,000 \mathrm{mg} / \mathrm{kg}$ when compared with those in the control. In addition, urine specific gravities were decreased in the males of these groups. These changes accompanied by an increase in water consumption, which was confirmed by an observation of water feeding bottles placed in the metabolic cages. Urinalysis showed some minor changes and was not suggestive of any toxic effects (data not shown).

The hematology data are presented in Table 2. At the end of treatment period, platelet counts were slightly lower in the $1,000 \mathrm{mg} / \mathrm{kg}$ treated females, but were statistically insignificant when compared with those in the control. No changes were observed in any of the hematology parameters of males at the end of treatment period. At the end of recovery period, there were statistically significant differences between the males of compound treated and
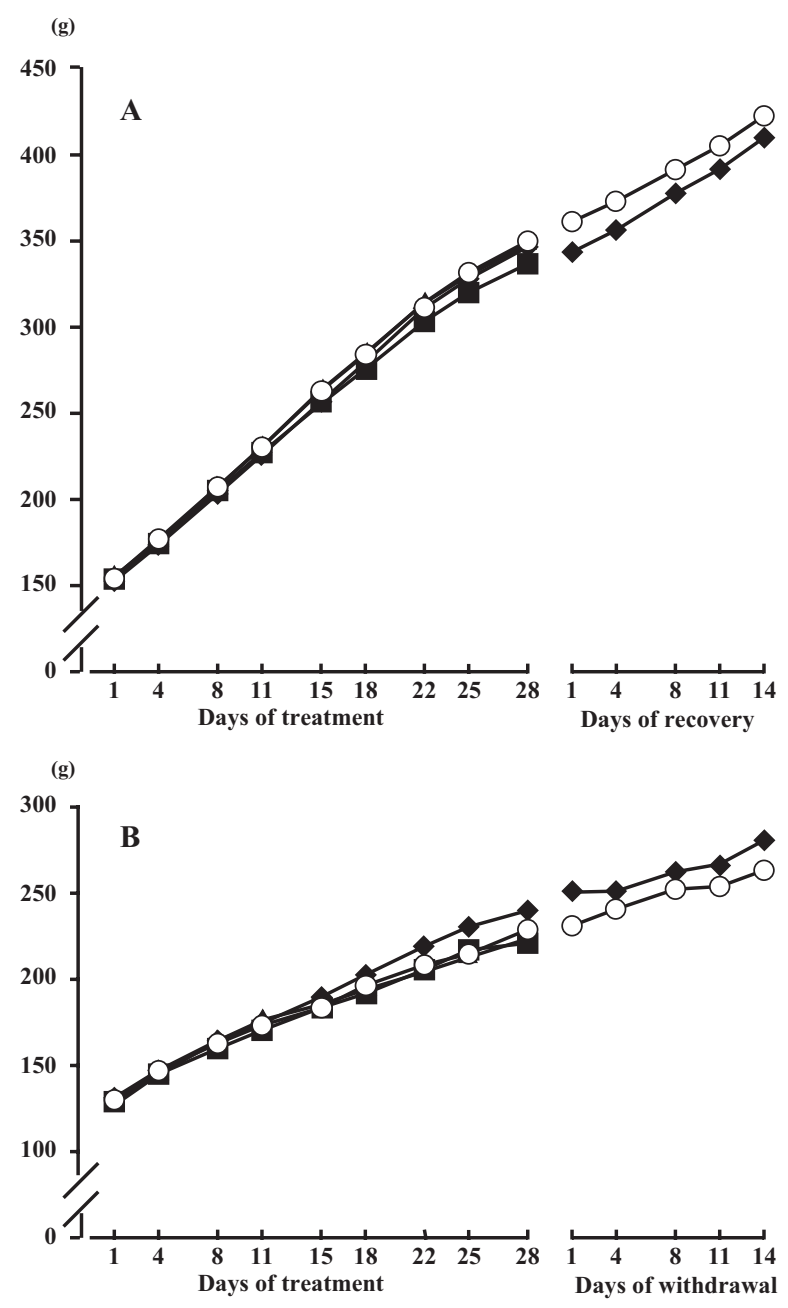

Fig. 1. Body weight changes of male (A) and female (B) rats treated orally with 4-methylbenzoic acid for 28-days at dose levels of $0(0), 100(\mathbf{\square}), 300(\boldsymbol{\Delta})$ and 1,000 mg/ $\mathrm{kg}$ /day $(\downarrow)$ in the repeated dose 28 -day oral toxicity study. Values during the treatment period represent the average for 5 animals in the 100 or $300 \mathrm{mg} / \mathrm{kg}$-treated group and for 10 animals in the 0 or $1,000 \mathrm{mg} / \mathrm{kg}$ treated group. Those during the recovery period represent the average for 5 animals. 
Screening of 4-methylbenzoic acid toxicities by OECD test guidelines

control groups, and were not suggestive any toxic effects.

The blood chemistry data are presented in Table 3 . At the end of treatment period, a moderate increase in AST activity and a slight decrease in total protein concentration were observed in the $1,000 \mathrm{mg} / \mathrm{kg}$-treated females, compared with those of control. In those data from males and those at the end of the recovery period from animals of both sexes, no significant differences were observed between the compound treated and control groups.

No significant differences from the control were observed in absolute organ weights or in organ weights relative to body weight in any of the compound treated groups of males (Table 4) or females (data not shown).

No abnormalities suggestive of any toxic effects were observed in any organs or tissues on gross examination at necropsy or histopathological examination (data not shown).

\section{Effects of repeated dosing in the reproduction/ developmental study}

As found in the 28-day study, temporary salivation was observed in a few animals given $1,000 \mathrm{mg} / \mathrm{kg}$ (data not shown). Except that, there were no clinical signs relating to the treatment.

Changes in body weight and food consumption of males are shown in Figs. 3 and 4, respectively. The compound did not affect body weight increase or food consumption, at any dose level.

Changes in body weight and food consumption of females are shown in Figs. 5 and 6, respectively. The compound did not affect body weights until Day 14 of gestation, at any dose level, while food consumption of the
$1,000 \mathrm{mg} / \mathrm{kg}$ treated group was slightly higher than that of the control at the beginning of the dosing. During Days 14-20 of gestation, however, maternal body weight gain was reduced in the $300 \mathrm{mg} / \mathrm{kg}$ or more treated groups, while the food consumption that was determined during this period (Day 14-15 of gestation) and body weight on

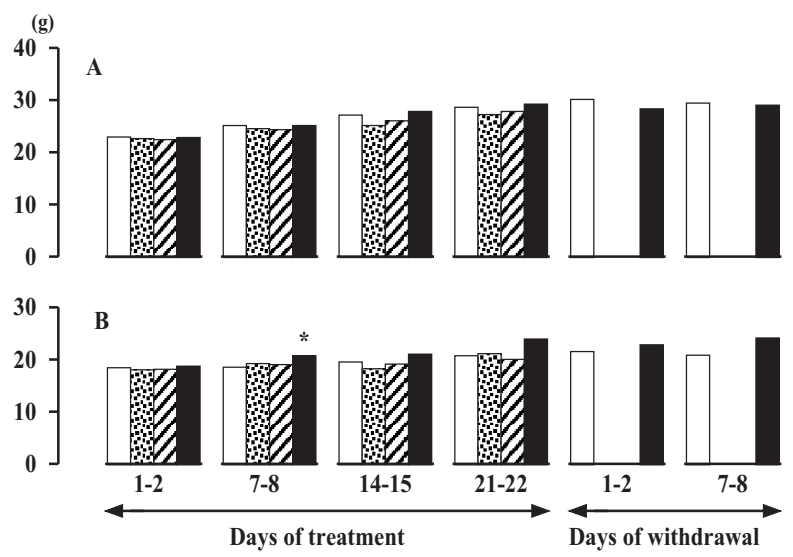

Fig. 2. Changes in food consumption of male (A) and female (B) rats treated orally with 4-methylbenzoic acid for 28-days at dose levels of 0 (open column), 100 (dashed column), 300 (hatched column) or $1,000 \mathrm{mg} / \mathrm{kg} /$ day (closed column) in the repeated dose 28-day oral toxicity study. Each column represents average for 5 animals in the 100 or $300 \mathrm{mg} / \mathrm{kg}$-treated group and for 10 animals in the 0 or $1,000 \mathrm{mg} / \mathrm{kg}$-treated group. During the recovery period, it represents the average for 5 animals. $*$ indicates significant difference from control at $\mathrm{p}<0.05$.

Table 1. Urinalysis of rats treated orally with 4-methylbenzoic acid in repeated dose 28-day oral toxicity study

\begin{tabular}{|c|c|c|c|c|c|c|}
\hline \multirow[b]{2}{*}{ Dose (mg/kg) } & \multicolumn{4}{|c|}{ On Day 23 of treatment } & \multicolumn{2}{|c|}{ On Day 9 of recovery } \\
\hline & 0 & 100 & 300 & 1,000 & 0 & 1,000 \\
\hline \multicolumn{7}{|c|}{ Number of animals (males/females) } \\
\hline & $10 / 10$ & $5 / 5$ & $5 / 5$ & $10 / 10$ & $5 / 5$ & $5 / 5$ \\
\hline \multicolumn{7}{|c|}{ Urinary volume $(\mathrm{ml} / 24 \mathrm{hr})$} \\
\hline $\begin{array}{r}\text { Males } \\
\text { Females }\end{array}$ & $\begin{array}{l}15.6 \pm 2.2 \\
11.7 \pm 3.5\end{array}$ & $\begin{array}{l}15.6 \pm 2.1 \\
11.9 \pm 3.4\end{array}$ & $\begin{array}{l}20.9 \pm 4.7^{*} \\
13.0 \pm 4.3\end{array}$ & $\begin{array}{l}23.8 \pm 4.7^{* *} \\
22.1 \pm 7.5^{* *}\end{array}$ & $\begin{array}{l}18.3 \pm 4.7 \\
13.3 \pm 2.8\end{array}$ & $\begin{array}{l}24.3 \pm 4.6 \\
18.3 \pm 5.6\end{array}$ \\
\hline \multicolumn{7}{|l|}{ Specific gravity } \\
\hline $\begin{array}{r}\text { Males } \\
\text { Females }\end{array}$ & $\begin{array}{l}1.058 \pm 0.008 \\
1.045 \pm 0.012\end{array}$ & $\begin{array}{l}1.051 \pm 0.007 \\
1.046 \pm 0.008\end{array}$ & $\begin{array}{l}1.043 \pm 0.011^{* *} \\
1.043 \pm 0.007\end{array}$ & $\begin{array}{l}1.045 \pm 0.006^{* *} \\
1.038 \pm 0.009\end{array}$ & $\begin{array}{l}1.056 \pm 0.009 \\
1.041 \pm 0.010\end{array}$ & $\begin{array}{l}1.038 \pm 0.006^{* *} \\
1.044 \pm 0.011\end{array}$ \\
\hline
\end{tabular}

Values represent average \pm S.D.

* and $* *$, significant difference from control at $\mathrm{p}<0.05$ and 0.01 , respectively. 
M. Shirota et al.

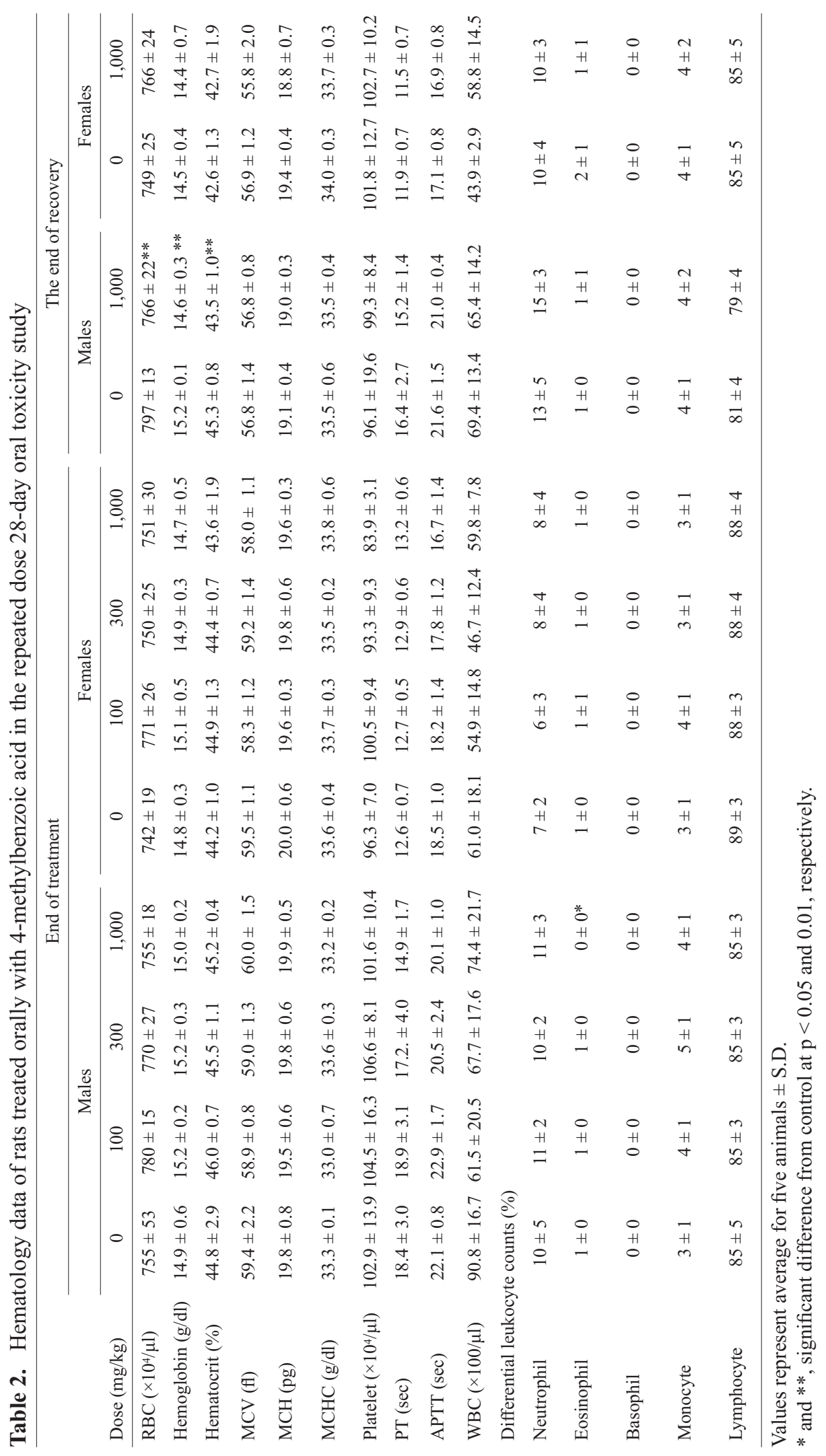

Vol. 33 No. 4 
Screening of 4-methylbenzoic acid toxicities by OECD test guidelines

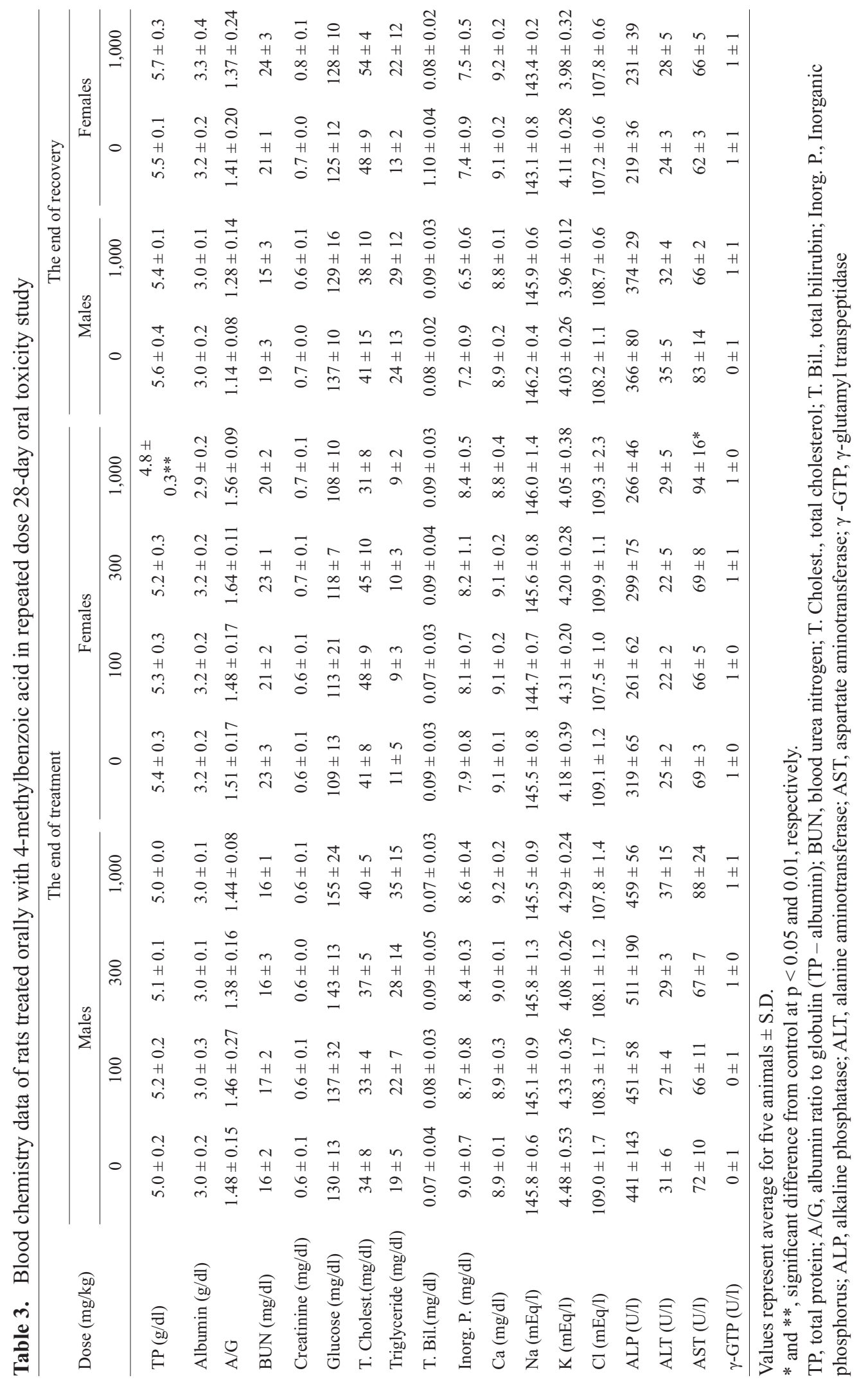

Vol. 33 No. 4 
Table 4. Organ weights of male rats treated orally with 4-methylbenzoic acid in repeated dose 28-day oral toxicity study

\begin{tabular}{|c|c|c|c|c|c|c|}
\hline \multirow[b]{2}{*}{ Dose (mg/kg) } & \multicolumn{4}{|c|}{ The end of treatment } & \multicolumn{2}{|c|}{ End of recovery } \\
\hline & 0 & 100 & 300 & 1,000 & 0 & 1,000 \\
\hline Number of animals & 5 & 5 & 5 & 5 & 5 & 5 \\
\hline Body weight (g) & $307.9 \pm 24.7$ & $301.4 \pm 32.0$ & $311.2 \pm 4.2$ & $311.8 \pm 30.7$ & $380.8 \pm 43.4$ & $370.2 \pm 15.1$ \\
\hline \multicolumn{7}{|l|}{ Absolute weight } \\
\hline Brain (g) & $1.92 \pm 0.07$ & $1.87 \pm 0.06$ & $1.89 \pm 0.07$ & $1.84 \pm 0.07$ & $1.92 \pm 0.11$ & $1.91 \pm 0.07$ \\
\hline Thymus (mg) & $587 \pm 86$ & $493 \pm 61$ & $537 \pm 95$ & $535 \pm 98$ & $423 \pm 42$ & $481 \pm 132$ \\
\hline Heart (g) & $1.10 \pm 0.09$ & $1.01 \pm 0.06$ & $1.00 \pm 0.05$ & $1.06 \pm 0.11$ & $1.21 \pm 0.15$ & $1.18 \pm 0.12$ \\
\hline Liver (g) & $9.62 \pm 0.99$ & $9.97 \pm 1.87$ & $9.73 \pm 0.46$ & $10.26 \pm 1.15$ & $10.95 \pm 2.00$ & $10.88 \pm 0.46$ \\
\hline Kidneys (g) & $2.39 \pm 0.20$ & $2.42 \pm 0.24$ & $2.43 \pm 0.17$ & $2.42 \pm 0.28$ & $2.72 \pm 0.30$ & $2.69 \pm 0.27$ \\
\hline Spleen (mg) & $707 \pm 192$ & $611 \pm 74$ & $643 \pm 85$ & $591 \pm 106$ & $743 \pm 114$ & $694 \pm 93$ \\
\hline Testes (g) & $2.69 \pm 0.01$ & $2.84 \pm 0.14$ & $3.00 \pm 0.28$ & $2.87 \pm 0.35$ & $2.94 \pm 0.16$ & $2.95 \pm 0.15$ \\
\hline Epididymides (g) & $0.67 \pm 0.04$ & $0.71 \pm 0.07$ & $0.70 \pm 0.02$ & $0.69 \pm 0.10$ & $0.91 \pm 0.06$ & $0.90 \pm 0.04$ \\
\hline Adrenal glands (mg) & $50.9 \pm 3.6$ & $45.6 \pm 5.6$ & $54.6 \pm 4.6$ & $47.1 \pm 6.3$ & $52.7 \pm 6.0$ & $55.8 \pm 6.8$ \\
\hline \multicolumn{7}{|c|}{$\underline{\text { Relative weight }(\mathrm{g} / 100 \mathrm{~g})}$} \\
\hline Brain & $0.63 \pm 0.04$ & $0.62 \pm 0.05$ & $0.61 \pm 0.03$ & $0.60 \pm 0.06$ & $0.51 \pm 0.03$ & $0.52 \pm 0.01$ \\
\hline Thymus & $0.19 \pm 0.02$ & $0.16 \pm 0.02$ & $0.17 \pm 0.03$ & $0.17 \pm 0.02$ & $0.11 \pm 0.02$ & $0.13 \pm 0.04$ \\
\hline Heart & $0.36 \pm 0.04$ & $0.34 \pm 0.03$ & $0.32 \pm 0.01$ & $0.34 \pm 0.02$ & $0.32 \pm 0.05$ & $0.32 \pm 0.03$ \\
\hline Liver & $3.12 \pm 0.16$ & $3.29 \pm 0.36$ & $3.13 \pm 0.17$ & $3.29 \pm 0.14$ & $2.87 \pm 0.32$ & $2.94 \pm 0.08$ \\
\hline Kidneys & $0.78 \pm 0.05$ & $0.80 \pm 0.05$ & $0.78 \pm 0.05$ & $0.78 \pm 0.06$ & $0.72 \pm 0.09$ & $0.73 \pm 0.07$ \\
\hline Spleen & $0.23 \pm 0.04$ & $0.20 \pm 0.02$ & $0.21 \pm 0.03$ & $0.19 \pm 0.03$ & $0.20 \pm 0.02$ & $0.19 \pm 0.02$ \\
\hline Testes & $0.88 \pm 0.13$ & $0.95 \pm 0.11$ & $0.97 \pm 0.09$ & $0.92 \pm 0.05$ & $0.78 \pm 0.07$ & $0.80 \pm 0.06$ \\
\hline Epididymides & $0.22 \pm 0.03$ & $0.24 \pm 0.02$ & $0.23 \pm 0.00$ & $0.22 \pm 0.02$ & $0.24 \pm 0.02$ & $0.24 \pm 0.02$ \\
\hline Adrenal glands & $0.017 \pm 0.002$ & $0.015 \pm 0.001$ & $0.018 \pm 0.002$ & $0.015 \pm 0.001$ & $0.014 \pm 0.001$ & $0.015 \pm 0.002$ \\
\hline
\end{tabular}

Values represent average \pm S.D.

the day of delivery (Day 0 of lactation) were higher in the $1,000 \mathrm{mg} / \mathrm{kg}$ treated group and in the $300 \mathrm{mg} / \mathrm{kg}$ or more treated groups, respectively, compared with those in the control. During the lactation period, maternal body weight gain and food consumption in the $1,000 \mathrm{mg} / \mathrm{kg}$ treated group were smaller than those in the control.

Terminal body weight and the weights of testes and epididymides of the male rats treated for 42 days are shown in Table 5. The compound did not affect the terminal body weight, at any dose level. While no differences were observed in the absolute or relative testicu- lar weights between the control and the compound treated groups, those of the epididymides were significantly lower in the $1,000 \mathrm{mg} / \mathrm{kg}$ treated group than in the control group. Histopathological examination revealed that there were lumens containing no or few spermatozoa, i.e., oligo/azoospermia, in the cauda epididymis in all the males of this group (Fig. 7), while none of their caput epididymis showed the same abnormality. In addition to this, the number of animals with cell debris in the cauda epididymal lumen was increased in this group, although it was statistically insignificant when compared with that in the 

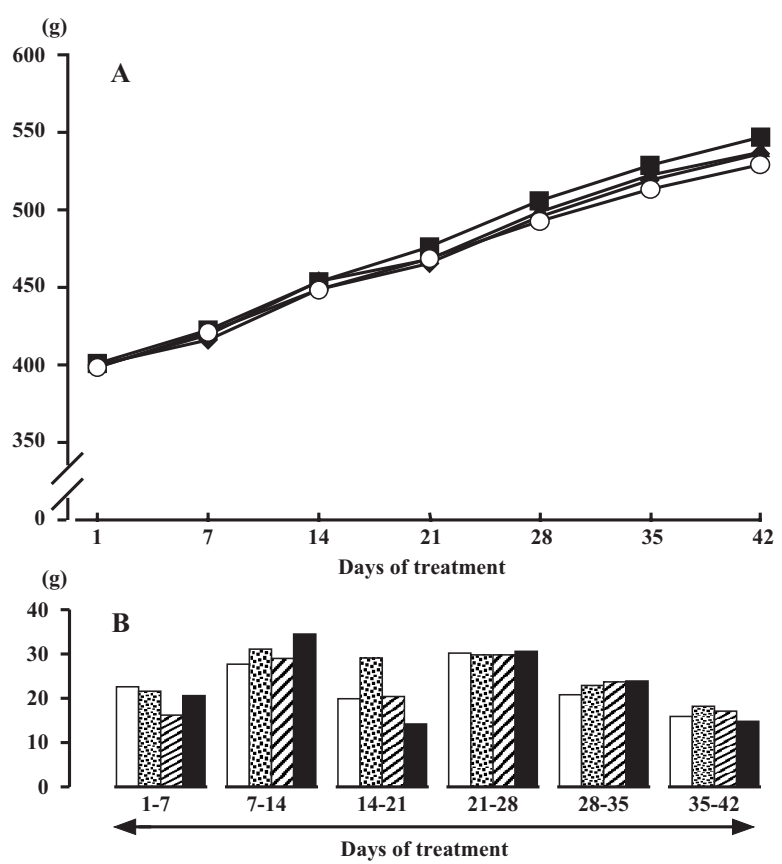

Fig. 3. Changes in body weight (A) and its gain (B) in male rats treated orally with 4-methylbenzoic acid for 42days at dose level of 0 ( $\mathrm{O}$ and open column), 100 ( and dashed column), 300 ( $\mathbf{\Delta}$ and hatched column) or $1,000 \mathrm{mg} / \mathrm{kg} /$ day ( and closed column) in the reproduction/developmental toxicity screening test. Each value represents the average for 13 animals.

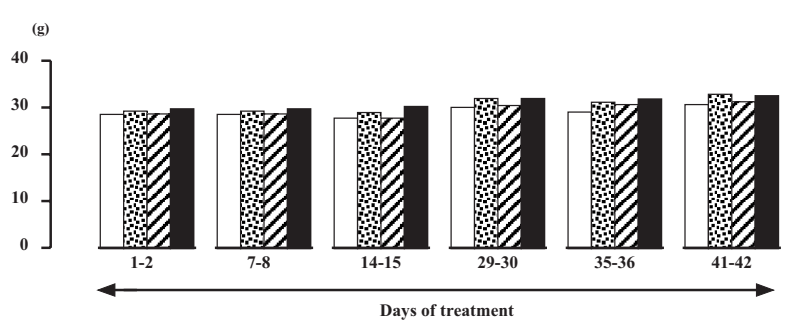

Fig. 4. Changes in food consumption of male rats treated orally with 4-methylbenzoic acid for 42-days at dose level of 0 (open column), 100 (dashed column), 300 (hatched column) or $1,000 \mathrm{mg} / \mathrm{kg} /$ day (closed column) in the reproduction/developmental toxicity screening test. Each column represents the average for 13 males.
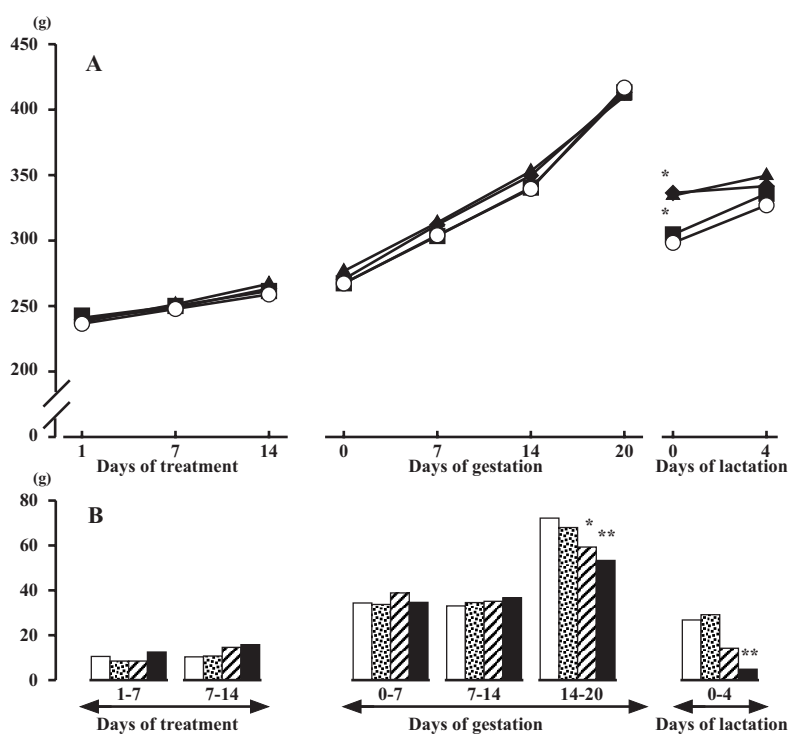

Fig. 5. Changes in body weight (A) and its gain (B) in female rats treated orally with 4-methylbenzoic acid at a dose level of 0 ( $\circ$ and open column), 100 ( $\square$ and dashed column), 300 ( $\boldsymbol{\Delta}$ and hatched column) or $1,000 \mathrm{mg} /$ $\mathrm{kg}$ /day ( and closed column) in the reproduction/developmental toxicity screening test. Administration of the compound was started 2 weeks prior to mating, and was continued through mating period and gestation period until 3 days after delivery. Each value during the pre-mating treatment period represents the average for 13 animals. That during the gestation and lactation periods represents the average for $9-13$ dams. $*$ and $* *$ indicate significant difference from control at $\mathrm{p}<0.05$ and 0.01 , respectively.

control. Males in the groups given $300 \mathrm{mg} / \mathrm{kg}$ or less did not show such abnormalities at any part of their epididymis (Table 6). In the testis, no abnormal findings related to the doses of the compound were observed.

\section{Reproductive performances in the reproduction/ developmental study}

Except one female in the control and one female in the $100 \mathrm{mg} / \mathrm{kg}$ treated groups, all the females revolved on a regular 4-day estrous cycle until mating (data not shown). Mating performance and dam data are shown in Table 7. None of indices for mating performance were different between the compound treated group and the control group. In addition, the number of corpora lutea, which represented the number of oocytes shed for impregnation, was not different between these groups. Thus, the compound did not affect the estrous cycle, ovulation or mating, at any dose level. 


\section{Shirota et al.}

Although all of the females that had copulated in the $100 \mathrm{mg} / \mathrm{kg}$ and less treated groups became pregnant, one such female and four such females in the $300 \mathrm{mg} / \mathrm{kg}$ and $1,000 \mathrm{mg} / \mathrm{kg}$ treated groups, respectively, did not become pregnant (Table 7). The Fisher's direct probability test indicated that there was a significant difference in the fertility between the control and the $1,000 \mathrm{mg} / \mathrm{kg}$ treated groups, which indicated that the compound disrupted fertility at $1,000 \mathrm{mg} / \mathrm{kg}$.

As shown in Table 7, pregnant females delivered live fetuses without differences in gestation length between the control and the compound treated groups. However, the implantation index and the number of pups born were significantly decreased in the $300 \mathrm{mg} / \mathrm{kg}$ or more treated groups. Furthermore, the numbers of pups alive on Days 0 and 4 of lactation were significantly smaller in the 1,000 $\mathrm{mg} / \mathrm{kg}$ treated group than those in the control. The other dam data, such as the birth index, the live birth index and the viability index on Day 4 of lactation, were not affected by the treatment. In addition, pup body weights at birth and on Day 4 of lactation and the sex ratios on these days were not affected by the treatment, in any group.

At necropsy of females, the uterus of the female that failed to become pregnant in the $300 \mathrm{mg} / \mathrm{kg}$ treated group showed ballooning and accumulation of cloudy fluid. Histopathology of the uterus revealed lumen dilatation and cellular infiltration of neutrophils in the epithelium and endometrial stroma, with edema in the endometrial stroma. In the $1,000 \mathrm{mg} / \mathrm{kg}$ treated group, however, no abnormality was observed in the reproductive organs of the four females that failed to become pregnant, either, at the gross necropsy or in the histopathological examina- tion of the ovary, while a moderate increase in atretic follicles was observed in one of the females. Including this case, there were no histopathological findings in the ovary related to doses of the compound (data not shown).

No abnormalities in the morphology or behavior of pups was noted in any group, except the following occasional cases in a single dam of the $1,000 \mathrm{mg} / \mathrm{kg}$ treated group: temporary cyanosis at birth and dilatation of renal pelvis at necropsy on Day 4 of lactation in one male pup.

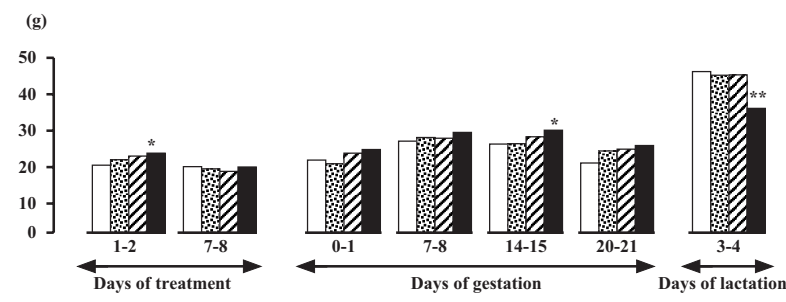

Fig. 6. Changes in food consumption of female rats treated orally with 4-methylbenzoic acid at dose levels of 0 (open column), 100 (dashed column), 300 (hatched column) or $1,000 \mathrm{mg} / \mathrm{kg} /$ day (closed column) in the reproduction/developmental toxicity screening test. Administration of the compound was started 2 weeks prior to mating, and was continued through mating period and gestation period until 3 days after delivery. Each value during the pre-mating treatment period represents the average for 13 females. That during the gestation and lactation periods represents the average for 9-13 dams. * and ** indicate significant difference from control at $\mathrm{p}<0.05$ and 0.01 , respectively.

Table 5. Weights of testes and epididymides in rats treated orally with 4-methylbenzoic acid for 42-days in reproduction/developmental toxicity screening test

\begin{tabular}{lcccc}
\hline Dose $(\mathrm{mg} / \mathrm{kg})$ & 0 & 100 & 300 & 1,000 \\
\hline Number of animals & 13 & 13 & 13 & 13 \\
Body weight $(\mathrm{g})$ & $527.9 \pm 37.8$ & $549.9 \pm 42.8$ & $541.6 \pm 26.6$ & $542.4 \pm 30.7$ \\
Absolute weight & & & $3.29 \pm 0.22$ & $3.31 \pm 0.17$ \\
Testes (g) & $3.37 \pm 0.24$ & $3.29 \pm 0.19$ & $1.24 \pm 0.07$ & $1.13 \pm 0.09^{* *}$ \\
Epididymides (g) & $1.28 \pm 0.08$ & $1.27 \pm 0.08$ & & $0.61 \pm 0.05$ \\
Relative weight (g/100 g) & & & $0.61 \pm 0.04$ & $0.21 \pm 0.02^{* *}$ \\
Testes & $0.64 \pm 0.07$ & $0.60 \pm 0.06$ & $0.23 \pm 0.01$ & $0.23 \pm 0.02$ \\
Epididymides & $0.24 \pm 0.03$ & 0.23 & & \\
\hline
\end{tabular}

Values represent average \pm S.D.

**, significant difference from control at $\mathrm{p}<0.01$. 

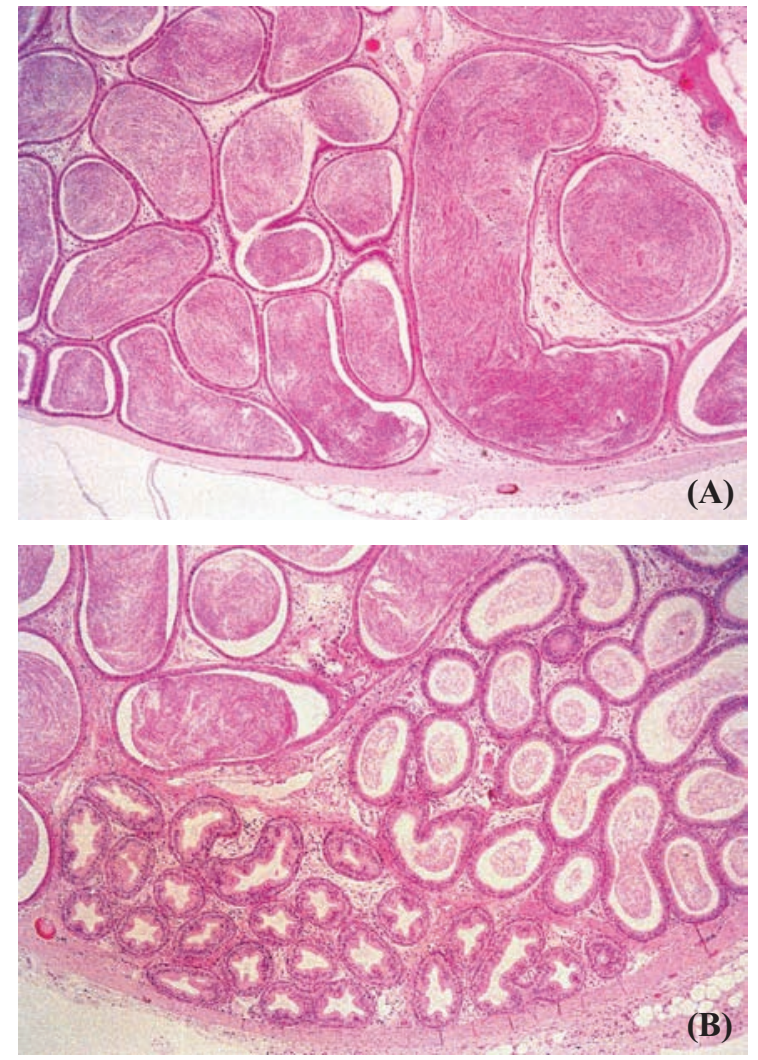

Fig. 7. Representative photographs of microscopic cross section of the cauda epididymis in male rats treated orally with 4-methylbenzoic acid at dose levels of 0 (A) or $1,000 \mathrm{mg} / \mathrm{kg} /$ day (B) in the reproduction study conducted under the OECD test guideline 421. Note that a few sperm are found in the lumen of the compound treated rats (B), and the epithelial height of the lumen becomes high. In contrast, the epithelial height of the lumen in the vehicle treated rats (A) becomes low by filling sperm in the lumen. Hematoxylin-eosin stain, $\mathrm{x} 36$.

\section{DISCUSSION}

Although the results obtained from the present studies are limited to short-term oral toxicity of 4-methylbenzoic acid, they indicate some toxicological properties of the compound. Namely, inconsistent effects of the compound on the epididymis indicate different toxicological potencies of the compound in male animals between the 28-day study and the reproduction/developmental study. While oral daily administration with $1,000 \mathrm{mg} / \mathrm{kg}$ of the compound reduced epididymal weight and caused oligo/ azoospermia in the cauda epididymal lumen in the reproduction/developmental study, the same dose of the compound did not cause such effects in the 28-day study.
Thus, the compound exerts adverse effects only on adult males when given for 42 days. Except that, repeated administration of $1,000 \mathrm{mg} / \mathrm{kg}$ of the compound led to similar results in males in both studies, such as a temporary salivation after dosing and no effects on body weight increase or food consumption. Therefore, the inconsistencies may be caused by differences in the timing or duration of administration.

The oligo/azoospermia seemed to develop at the distal part of the epididymis, since no abnormalities were observed in the caput epididymis and since there were normal lumens in the cauda epididymis. As reported by Robaire et al. (2006), spermatozoa enter the epididymis with testicular fluid and progress downward toward the vas deferens by smooth muscle contraction. In the reproduction/developmental study, the oligo/azoospermia developed where mature spermatozoa are stored. Spermatozoa are found in seminiferous tubules at the time of puberty, approximately 6 weeks of age in the rat, and it takes at least 10 or 15 days to reach the cauda epididymis (Sommer et al., 1996; Robaire et al., 2006). We have confirmed previously that spermatozoa are found in the cauda epididymis at 56 days of age at the youngest in the Sprague-Dawley derived inbred rats, which attain puberty at a comparable range of ages to Sprague-Dawley rats (Sato et al., 2002). Since the compound was administered for 4 weeks in the 28-day study, from 5 weeks of age, the compound could influence epididymal spermatozoa for a short period. The storage period of spermatozoa in the cauda epididymis is estimated to be one week. In contrast, the compound was administered from 10 weeks of age in the reproduction/developmental study and could influence spermatozoa in all segments of the epididymis for 42 days. The inconsistent adverse effects of the compound on the cauda epididymis between the studies could be explained by either, the timing or the duration, of the administration.

Although the results obtained from the reproduction/ developmental study seemed to suggest epididymal spermatozoa as the toxicological target of the compound, 4methylbenzoic acid could influence spermatozoa function during their transit to the cauda epididymis. It has been reported that spermatozoa become functionally mature during transit to the distal part of the epididymis, while they are protected from oxidative stress and harmful xenobiotics by the blood-epididymis barrier and different types of antioxidant enzymes in each segment of the epididymis (Tengpwski et al., 2007; Kim et al., 2004; Robaire et al., 2006). Therefore, detailed investigation of the effects of the compound on production, function and transition of spermatozoa is required. 
M. Shirota et al.

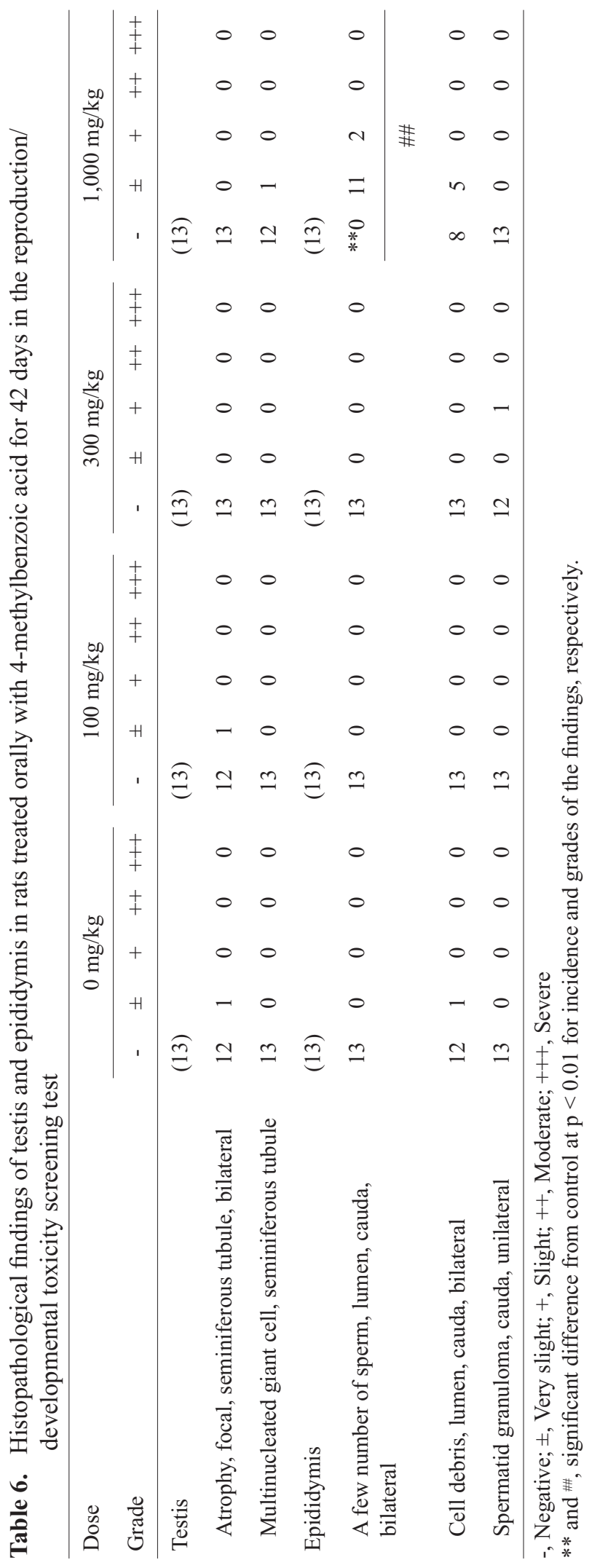

Vol. 33 No. 4 
Screening of 4-methylbenzoic acid toxicities by OECD test guidelines

Table 7. Mating performances and dam data of rats treated orally with 4-methylbenzoic acid in the reproduction/ developmental toxicity screening test

\begin{tabular}{|c|c|c|c|c|}
\hline Dose (mg/kg) & 0 & 100 & 300 & 1,000 \\
\hline \multicolumn{5}{|l|}{ Mating performance } \\
\hline Copulated pairs/Co-housed pairs (\%) & $13 / 13(100)$ & $13 / 13(100)$ & $12 / 13(92.3)$ & $13 / 13(100)$ \\
\hline Pregnant females/Copulated pairs (\%) & $13 / 13(100)$ & $13 / 13(100)$ & $11 / 12(91.7)$ & $9 / 13(69.2)^{*}$ \\
\hline Pairing days until copulation a) & $3.0 \pm 3.4$ & $2.4 \pm 1.3$ & $2.4 \pm 1.3$ & $3.2 \pm 3.3$ \\
\hline Number of estrus revolved until copulation a) & $1.0 \pm 0.0$ & $1.0 \pm 0.0$ & $1.0 \pm 0.0$ & $1.1 \pm 0.3$ \\
\hline \multicolumn{5}{|l|}{ Dam data } \\
\hline Number of pregnant females with live pups & 13 & 13 & 11 & 9 \\
\hline Gestation length in days a) & $22.3 \pm 0.5$ & $22.5 \pm 0.5$ & $22.3 \pm 0.5$ & $22.8 \pm 0.4$ \\
\hline Number of corpora lutea a) & $16.2 \pm 1.3$ & $15.7 \pm 1.3$ & $16.2 \pm 1.5$ & $15.9 \pm 1.3$ \\
\hline Number of implantation sites a) & $16.0 \pm 1.3$ & $15.4 \pm 1.0$ & $14.0 \pm 2.4$ & $12.1 \pm 5.3$ \\
\hline Implantation index a, b) & $99.1 \pm 2.2$ & $98.2 \pm 3.5$ & $86.5 \pm 12.6^{* *}$ & $75.9 \pm 32.6^{*}$ \\
\hline \multicolumn{5}{|l|}{ Day 0 of lactation } \\
\hline Number of pups born a) & $15.2 \pm 1.4$ & $14.1 \pm 1.8$ & $12.5 \pm 2.1 * *$ & $10.2 \pm 5.1^{* *}$ \\
\hline Delivery index a,c) & $94.7 \pm 5.2$ & $91.4 \pm 8.5$ & $90.1 \pm 8.4$ & $82.9 \pm 19.5$ \\
\hline Number of pups alive & $14.3 \pm 1.7$ & $14.1 \pm 1.8$ & $12.3 \pm 2.2$ & $10.0 \pm 5.0^{*}$ \\
\hline Birth index a, d) & $89.7 \pm 10.4$ & $91.4 \pm 8.5$ & $88.0 \pm 9.1$ & $81.5 \pm 19.8$ \\
\hline Live birth index ${ }^{a, e)}$ & $94.8 \pm 10.5$ & $100 \pm 0$ & $97.6 \pm 4.2$ & $98.2 \pm 3.7$ \\
\hline Sex ratio a, f) & $54.1 \pm 14.3$ & $51.9 \pm 12.8$ & $46.6 \pm 13.8$ & $59.8 \pm 17.7$ \\
\hline \multicolumn{5}{|l|}{ Day 4 of lactation } \\
\hline Number of pups alive a) & $14.3 \pm 1.7$ & $13.9 \pm 1.8$ & $12.3 \pm 2.2$ & $9.7 \pm 5.3 *$ \\
\hline Viability index a) & $100 \pm 0$ & $99.0 \pm 3.7$ & $100 \pm 0$ & $88.1 \pm 33.1$ \\
\hline Sex ratio a, f) & $54.1 \pm 14.3$ & $52.3 \pm 12.3$ & $46.6 \pm 13.8$ & $54.4 \pm 9.5$ \\
\hline \multicolumn{5}{|l|}{ Pups weight on Day 0 of lactation (g/pup) } \\
\hline Male a) & $6.8 \pm 0.4$ & $7.0 \pm 0.5$ & $7.0 \pm 0.5$ & $6.7 \pm 0.3$ \\
\hline Female a) & $6.5 \pm 0.4$ & $6.7 \pm 0.6$ & $6.6 \pm 0.5$ & $6.4 \pm 0.5$ \\
\hline \multicolumn{5}{|l|}{ Pups weight on Day 4 of lactation (g/pup) } \\
\hline Male a) & $10.7 \pm 1.0$ & $11.2 \pm 1.1$ & $11.8 \pm 1.2$ & $11.1 \pm 0.9$ \\
\hline Female a) & $10.3 \pm 1.0$ & $10.7 \pm 1.1$ & $11.2 \pm 1.3$ & $10.5 \pm 0.9$ \\
\hline
\end{tabular}

a) Average \pm S.D.; b) (Number of implantation sites/Number of corpora lutea) $\times 100, \%$; c) (Number of pups born/Number of implantation sites) $\times 100, \%$; d) (Number of live pups on Day 0 of lactation/Number of implantation sites) $\times 100$, \%; e) (Number of live pups on Day 0 of lactation/Number of pups born) $\times 100, \%$; f) (Number of live male pups/Number of live pups) $\times 100, \%$ $*$ and $* *$, significant difference from control at $\mathrm{p}<0.05$ and 0.01 , respectively.

The reproduction/developmental study revealed that the compound reduced fertility of animals within a relatively short period of dosing, since the four males that failed to impregnate in the $1,000 \mathrm{mg} / \mathrm{kg}$ treated group had copulated on Days 16, 19, 19 and 28 of treatment. Furthermore, the compound increased preimplantation loss and decreased implantation index at a lower dose level than that caused oligo/azoospermia. It is not clear whether the reduced fertility and the increase in the preimplantation loss are consequent effects of the compound on the epididymal spermatozoa or are effects on such as fertilization, early embryonic development or implantation, although the results obtained from the reproduction/developmental study indicate no adverse effects on female reproductive function until mating.

Because mating was done within the same dose groups 
in the reproduction/developmental study, it was impossible to determine the target sex of the compound. The reproductive toxicities of several compounds related to 4methylbenzoic acid have been studied. A feeding administration of $p$-nitrobenzoic acid (CAS No. 62-23-7) (NTP, 1994) and m-nitrobenzoic acid (CAS No. 121-92-6) has been found to reduce the number of offspring in continuous breeding studies in CD-1 mice (NTP/NIEHS, 1997). Crossover mating conducted in these studies revealed that female reproduction is more sensitive than male reproduction. On the other hand, 3-methylbenzoic acid (CAS No. 99-04-7) and 4-hydroxybenzoic acid (CAS No. 9996-7) did not show any reproductive toxicity in studies conducted under a protocol similar to the reproduction/ developmental study (Nagao et al., 1997; Yamamoto et al., 1999), although estrogenic potency of 4-hydroxybenzoic acid was observed in a uterotrophic assay (Lemini et al., 1997). Detailed investigation, such as crossover mating, quantification and qualification of spermatozoa, may elucidate the characteristics of the reproductive toxicity of 4-methylbenzoic acid.

In the reproduction/developmental study, maternal body weight was reduced during the latter period of gestation at the dose levels of $300 \mathrm{mg} / \mathrm{kg}$ or more, whereas it did not affect female body weight, at any dose level, in the 28-day study. It is clear, however, that the decrease in the maternal body weight was not caused by the direct toxicity of the compound. It was caused by a small litter size resulting from a reduced number of implantations, as discussed above, since fetal weight in the uterus greatly contributes to maternal body weight during the latter period of gestation. Comparable or greater food consumption during this period and greater body weight after parturition in dams of these groups also indicate no adverse effects of the compound on the maternal animals. Decreases in food consumption and body weight gain during the lactation period in the $1,000 \mathrm{mg} / \mathrm{kg}$ treated group might be physiological changes due to a smaller demand of nutrition for the small litter size.

In the males, urine specific gravity was decreased in the $300 \mathrm{mg} / \mathrm{kg}$ or more treated groups. The change, however, was reflected the increase in urine volume due to increased water consumption, and did not accompany morphological alterations or functional impairments of their kidneys. Therefore, the change was judged as a physiological response. Except for the effects on the epididymis in the reproduction/development study, no systemic effect of the compounds was observed in the males of both studies.

In the females, a slight increase in food consumption was observed in the $1,000 \mathrm{mg} / \mathrm{kg}$ treated group at the beginning of dosing in both studies. In the 28-day study, increase in AST activity and decrease in serum total protein concentration were observed in the $1,000 \mathrm{mg} / \mathrm{kg}$ treated females. Since increase in AST activity has been reported as an effect of 3-methylbenzoic acid (CAS No. 99-04-7) in the study noted above (Yamamoto et al., 1999), 4-methylbenzoic acid may slightly alter female AST activity. Thus, the compound affected females at the dose level of 1,000 mg/kg. However, all these changes did not accompany structural changes and changes in organ weights. Therefore, repeated dosing of the compound may affect females at the dose level of 1,000 mg/ $\mathrm{kg}$, but may not affect adversely, at any dose level.

From these results, the no-observed-effect-level (NOEL) for reproductive toxicity is considered to be 100 $\mathrm{mg} / \mathrm{kg}$, whereas $1,000 \mathrm{mg} / \mathrm{kg}$ did not show any effect on neonates. That for repeated dose toxicity is considered to be $300 \mathrm{mg} / \mathrm{kg}$ for male and female rats in the both studies, but toxic effects on the epididymis differed between the studies. Thus, 4-methylbenzoic acid has a potential for reproductive toxicity and deserves further study, taking the exposure state into consideration, as discussed above, since it has been reported that the reproduction/ developmental study provides screening information but does not provide a complete characterization and evaluation of reproductive or developmental toxicity (Gelbke et al., 2004).

\section{ACNOWLEDGMENT}

This study was partially supported by the Ministry of Health, Labour and Welfare as a part of programs of safety examination for existing chemical substances.

\section{REFERENCES}

Gelbke, H.P., Fleig, H., Meder, M. and German Chemical Industry Association. (2004): SIDS reprotoxicity screening test update: testing strategies and use. Regul. Toxicol. Pharmacol., 39, 8186.

Kim, E., Nishimura, H., Iwase, S., Yamagata, K., Kashiwabara, S. and Baba, T. (2004): Synthesis, processing, and subcellular localization of mouse ADAM3 during spermatogenesis and epididymal sperm transport. J. Reprod. Dev. 50, 571-578.

Lemini, C., Silva, G., Timossi, C., Luque, D., Valverde, A., González-Martínez, M., Hernández, A., Rubio-Póo, C., Chávez Lara, B. and Valenzuela F. (1997): Estrogenic effects of phydroxybenzoic acid in CD1 mice. Environ. Res., 75, 130-134.

Mineshita, T., Toteno, I., Yui, Y., Furukawa, S. and Wakabayashi, K. (1978): Acute toxicity study of p-toluic acid, The Clinical Report, 12, 1893-1904.

Nagao, T., Kuwagata, M., Kato, H. and Miyahara, T. (1997): Combined repeat dose and reproductive/developmental toxicity 
Screening of 4-methylbenzoic acid toxicities by OECD test guidelines

screening test of hydroxybenzoic acid in rats. Toxicity Testing Reports of Environmental Chemicals 5, 251-257.

National Toxicology Program (1994): NTP Toxicology and Carcinogenesis Studies of p-Nitrobenzoic Acid (CAS No. 62-23-7) in F344/N Rats and B6C3F1 Mice (Feed Studies). Natl. Toxicol. Program Tech. Rep. Ser. 442,1-306.

National Toxicology Program / National Institute of Environmental Health Sciences (1997): Reproductive toxicology. m-Nitrobenzoic acid. Environ. Health Perspect., 105 (Suppl. 1), 325-326.

OECD (1997a): OECD Guideline for the Testing of Chemicals 407, "Repeated Dose 28-day Oral Toxicity Study in Rodents" adopted on 27 July 1995.

OECD (1997b): OECD Guideline for the Testing of Chemicals 421, "Reproduction/Developmental Toxicity Screening Test" adopted on 27 July 1995.

OECD (2004): The 2004 OECD list of high production volume chemicals.

Robaire, B., Hinton, B. T. and Orgebin-Crist, M-C. (2006); The epididymis. In Knobil and Neill's Physiology of Reproduction, $3^{\text {rd }}$ ed. (Neill, J. D. ed.) pp.1071-1148, Academic Press, USA and
UK.

Sato, M., Ohta, R., Kojima, K., Shirota, M., Koibuchi, H., Asai, S., Watanabe, G. and Taya, K. (2002): A comparative study of puberty and plasma gonadotropin and testicular hormone levels in two inbred strains of Hatano rats. J. Reprod. Dev., 48, 111119.

Sommer, R. J., Ippolito, D. L., and Peterson, R.E.(1996): In utero and lactational exposure of the male Holtzman rat to 2,3,7,8-tetrachlorodibenzo-p-dioxin: decreased epididymal and ejaculated sperm numbers without alterations in sperm transit rate. Toxicol. Appl. Pharmacol., 140, 146-153.

Tengowski, M.W., Feng, D., Sutovsky, M. and Sutovsky, P. (2007); Differential expression of genes encoding constitutive and inducible 20S proteasomal core subunits in the testis and epididymis of theophylline- or 1,3-dinitrobenzene-exposed rats. Biol. Reprod., 76,149-163.

Yamamoto, Y., Ito, Y., Nosa, A., Ito, M., Akagi, H. and Hoshi, F. (1999): Combined repeat dose and reproductive/developmental toxicity screening test of 3-methylbenzoic acid in rats. Toxicity Testing Reports of Environmental Chemicals 7, 302-308. 